\title{
Towards an FDIR Software Fault Tree Library for Onboard Computers
}

IEEE Aerospace 2020

Sascha Müller, Kilian Höflinger, Michal Smisek, Andreas Gerndt

DLR German Aerospace Center

Institute of Simulation and Software Technology

Software for Space Systems and Interactive Visualization

Braunschweig

March 13, 2020

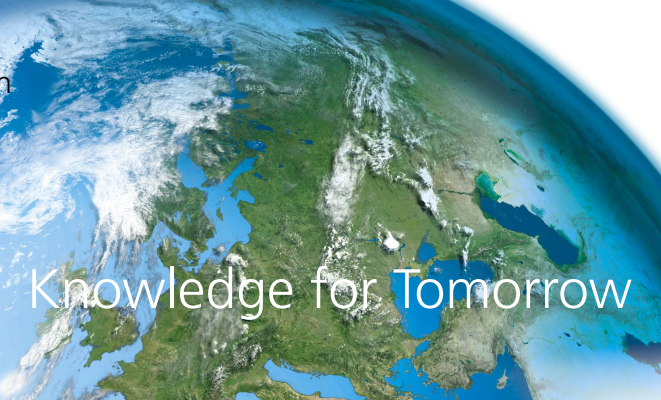




\section{Outline}

7 FDIR Analysis with Fault Trees

$\neg$ Problem Statement

$>$ FDIR Software Fault Tree Library

7 Dependability Quality Model

7 Use Case: MMX

7 Results

$\rightarrow$ Conclusion 


\section{Fault Detection, Isolation and Recovery}

\section{FDIR}

Even well designed systems cannot avoid the existence of faults

$>$ But not every fault is a failure

$>$ FDIR tries to prevent faults from turning into failures 


\section{FDIR}

Even well designed systems cannot avoid the existence of faults

$>$ But not every fault is a failure

$>$ FDIR tries to prevent faults from turning into failures

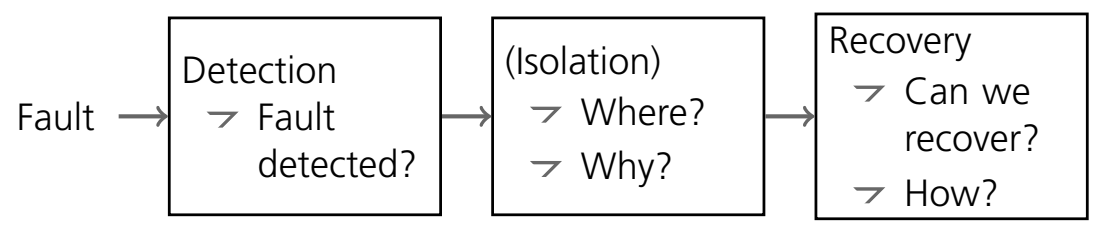




\section{On-Board Computing in Space}

7 Demand for powerful on-board computing is increasing (navigation, loss-less compression, artificial intelligence) due to communication limitations 


\section{High Performance On-Board Computers and FDIR}

\section{On-Board Computing in Space}

$>$ Demand for powerful on-board computing is increasing (navigation, loss-less compression, artificial intelligence) due to communication limitations

$>$ Idea: Use Common of the shelf components 


\section{High Performance On-Board Computers and FDIR}

\section{On-Board Computing in Space}

$>$ Demand for powerful on-board computing is increasing (navigation, loss-less compression, artificial intelligence) due to communication limitations

$>$ Idea: Use Common of the shelf components

$>$ But: Space is a harsh environment

$\rightarrow$ Radiation effects

$>$ Limited room for human intervention 


\section{High Performance On-Board Computers and FDIR}

\section{On-Board Computing in Space}

$>$ Demand for powerful on-board computing is increasing (navigation, loss-less compression, artificial intelligence) due to communication limitations

7 Idea: Use Common of the shelf components

$>$ But: Space is a harsh environment

$\rightarrow$ Radiation effects

7 Limited room for human intervention

Need FDIR to provide stable operation

How to plan and assess the FDIR concept in the design phase? 


\section{Modeling the $\mathrm{F}$ in FDIR}

\section{Fault Model}

Relationship between basic faults and how they lead to failures

$>$ Failure Modes and Effects Analysis (FMECA)

$\rightarrow$ Reliability Block Diagrams

7 Markov Modeling

7 Fault Tree Analysis (FTA)

7 ... and many more 


\section{Basics of Fault Trees}

\section{Fault Tree}

How do faults propagate through components?

$>$ Propagation model using gates (AND, OR, SPARE, PAND, etc.) 


\section{Basics of Fault Trees}

\section{Fault Tree}

How do faults propagate through components?

$>$ Propagation model using gates (AND, OR, SPARE, PAND, etc.)

$>$ Leaves: Basic Events (Resolution of analysis) 


\section{Basics of Fault Trees}

\section{Fault Tree}

How do faults propagate through components?

$>$ Propagation model using gates (AND, OR, SPARE, PAND, etc.)

$>$ Leaves: Basic Events (Resolution of analysis)

$>$ Basic Events can have failure rates for quantitative evaluation 


\section{Basics of Fault Trees}

\section{Fault Tree}

How do faults propagate through components?

$>$ Propagation model using gates (AND, OR, SPARE, PAND, etc.)

$>$ Leaves: Basic Events (Resolution of analysis)

$>$ Basic Events can have failure rates for quantitative evaluation

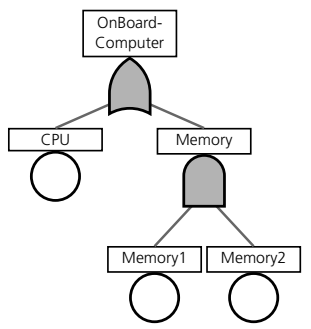




\section{Basics of Fault Trees}

\section{Fault Tree}

How do faults propagate through components?

$>$ Propagation model using gates (AND, OR, SPARE, PAND, etc.)

$>$ Leaves: Basic Events (Resolution of analysis)

$>$ Basic Events can have failure rates for quantitative evaluation

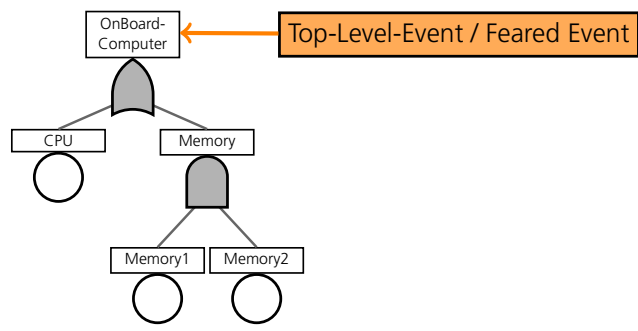




\section{Basics of Fault Trees}

\section{Fault Tree}

How do faults propagate through components?

$>$ Propagation model using gates (AND, OR, SPARE, PAND, etc.)

$>$ Leaves: Basic Events (Resolution of analysis)

$>$ Basic Events can have failure rates for quantitative evaluation

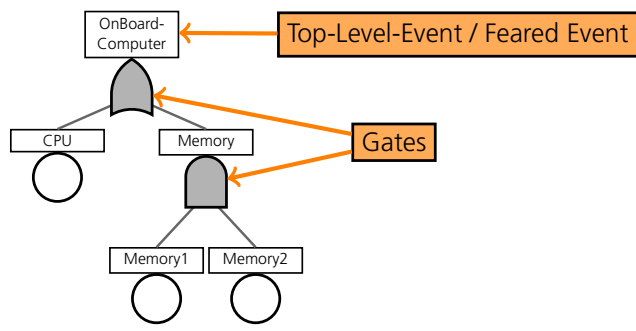




\section{Basics of Fault Trees}

\section{Fault Tree}

How do faults propagate through components?

$>$ Propagation model using gates (AND, OR, SPARE, PAND, etc.)

$>$ Leaves: Basic Events (Resolution of analysis)

$>$ Basic Events can have failure rates for quantitative evaluation

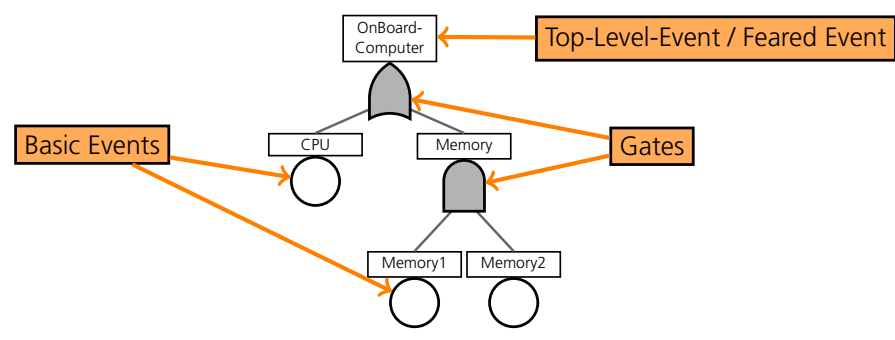




\section{Fault Trees and RAMS}

Fault Model

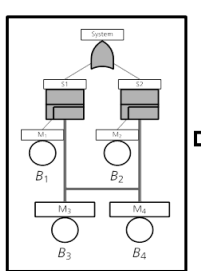

Mathematical Model

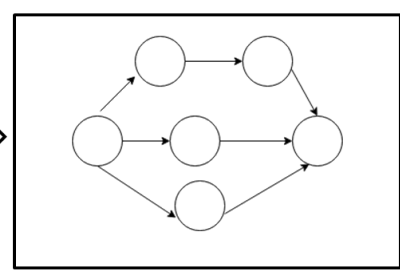

Figure: Fault Tree Evaluation
RAMS Metrics

ModeliChecking

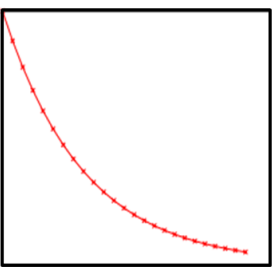

Reliability 


\section{Problem Statement}

Fault trees provide great analysis benefits but...

$>$ also require a lot of modeling effort!

$\checkmark$ need to be redone mostly from scratch for each new system! 


\section{Problem Statement}

Fault trees provide great analysis benefits but...

$>$ also require a lot of modeling effort!

$\checkmark$ need to be redone mostly from scratch for each new system!

Can we develop..

7 a generic FDIR software library

7 generic fault tree models for FDIR Software library?

7 a methodology to easily generate fault trees incorporating calls to the library? 


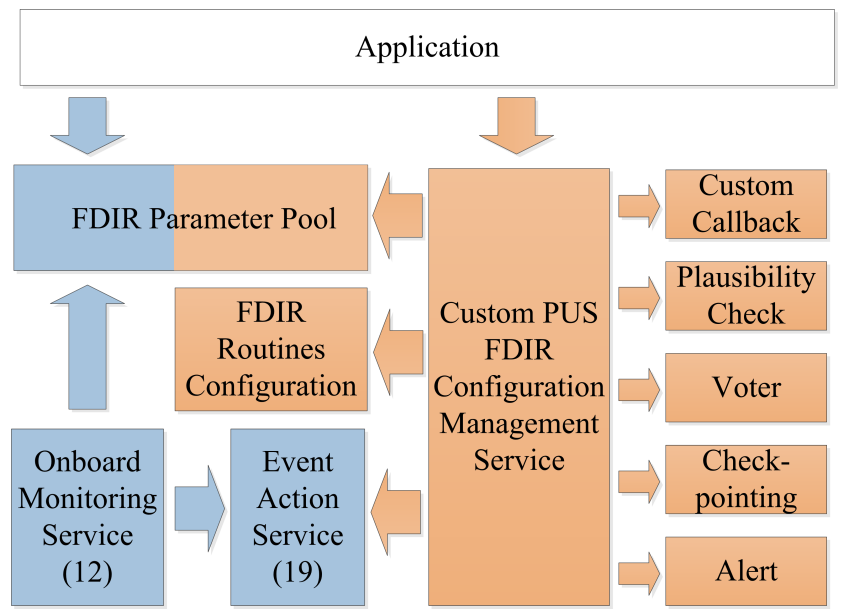

Figure: FDIR C++ Library Architecture 


\section{Fault Tree Generation}

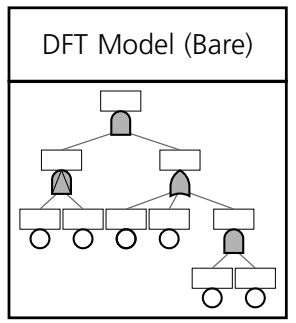

Figure: Generation process with DFT model and service library models. 


\section{Fault Tree Generation}
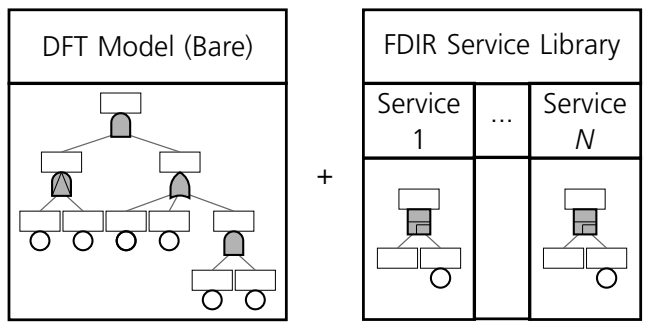

Figure: Generation process with DFT model and service library models. 


\section{Fault Tree Generation}

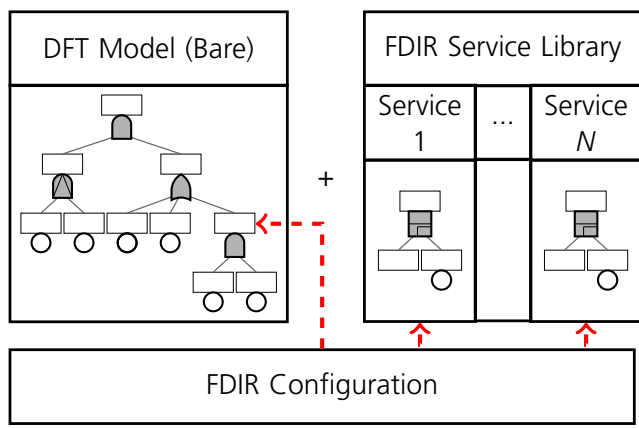

Figure: Generation process with DFT model and service library models. 


\section{Fault Tree Generation}

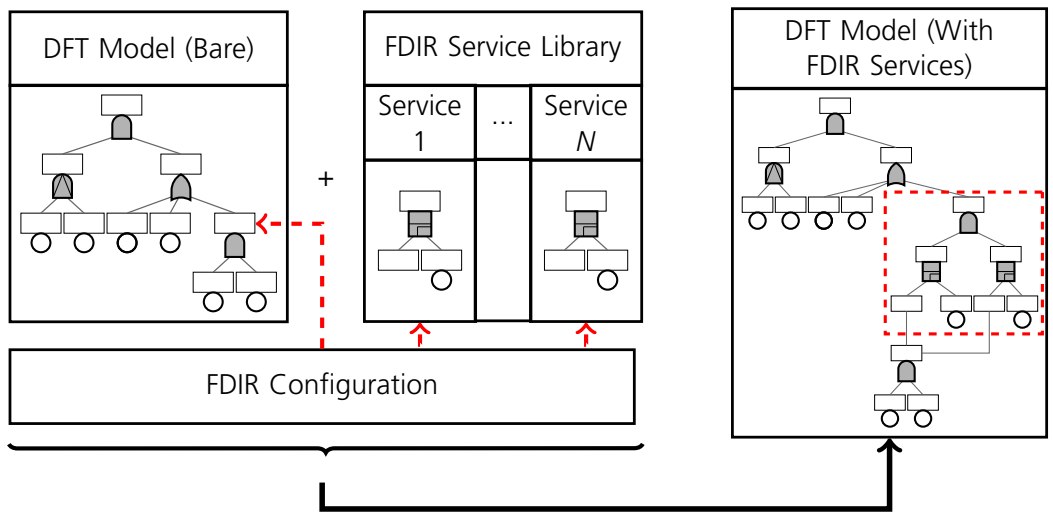

Figure: Generation process with DFT model and service library models. 


\section{Quality Model}

\begin{tabular}{|c|c|c|c|} 
Characteristic & $\begin{array}{c}\text { Sub } \\
\text { Characteristic }\end{array}$ & Metric & Threshold \\
\hline \hline \multirow{2}{*}{ Reliability } & $\begin{array}{c}\text { Reliability } \\
\text { Evidence }\end{array}$ & $\begin{array}{c}\text { Reliability } \\
\text { after t }\end{array}$ & $\begin{array}{c}>95 \% \text { in } \\
50 \mathrm{~d}\end{array}$ \\
& $\begin{array}{c}\text { Structural } \\
\text { Coverage }\end{array}$ & $100 \% *$ \\
\hline \multirow{2}{*}{$\begin{array}{c}\text { Maintain- } \\
\text { ability }\end{array}$} & Complexity & $\begin{array}{c}\text { Cyclomatic } \\
\text { Complexity }\end{array}$ & $<12^{*}$ \\
\hline \multirow{2}{*}{ Availabilty } & Modularity & $\begin{array}{c}\text { Modular } \\
\text { Coupling }\end{array}$ & $<4^{*}$ \\
\hline & Evidence & MTTF & $>50 \mathrm{~d}$ \\
\hline
\end{tabular}

Figure: Quality mode using factor-criteria-metric model (Based on ECSS) 


\section{Implementation - Virtual Satellite 4 FDIR}

\section{Virtual Satellite}

$>$ Model Based Systems Engineering tool

7 FDIR Extension supporting Model-Based Fault Tree Analysis

$\rightarrow$ https://github.com/virtualsatellite 


\section{Implementation - Virtual Satellite 4 FDIR}

\section{Virtual Satellite}

$>$ Model Based Systems Engineering tool

$>$ FDIR Extension supporting Model-Based Fault Tree Analysis

$>$ https://github.com/virtualsatellite
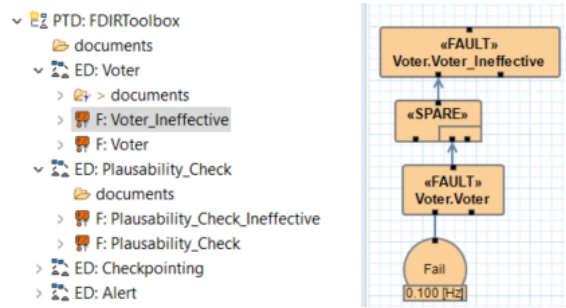

Figure: Excerpt of the library in VirSat 


\section{Mission}

7 Martian Moon eXploration (Phobos)

$\rightarrow$ Carries rover exploration with various payloads

$\rightarrow$ Limited communication windows and delays: High degree of autonomy required

$>$ Single OBC based on COTS components 
Mission

7 Martian Moon eXploration (Phobos)

$>$ Carries rover exploration with various payloads

$\rightarrow$ Limited communication windows and delays: High degree of autonomy required

$>$ Single OBC based on COTS components

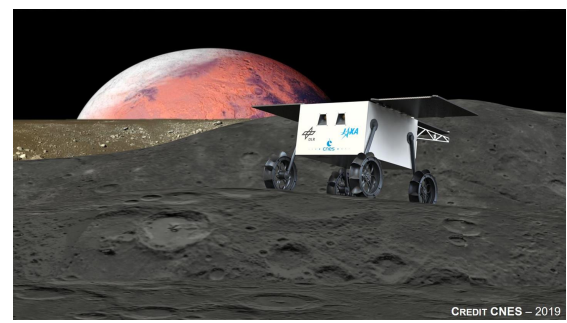

Figure: Artist impression of the MMX rover on Phobos (credit: CNES) 
Services

Feared Events

7 Loss of Position Service

$\rightarrow$ Loss of Obstacle Detection Service

DLR 


\section{Services}

Feared Events

7 Loss of Position Service

$\rightarrow$ Loss of Obstacle Detection Service

Applications

7 Rectification: Reverses lens distortions

7 Depth Image Computation: Computes disparity image and depth image

7 Visual Odometry: Measures rover's egomotion

7 Obstacle Detection Algorithm: Utilizes camera images and depth images to detects obstacles and terrain featuress 


\section{Basic Events}

\section{Main Events}

7 Short mission duration (50 days): Focus on short-term effects rather than long-term accumulation effects

$>$ Main focus: Single-Event Effects (SEE) 


\section{Basic Events}

\section{Main Events}

$>$ Short mission duration (50 days): Focus on short-term effects rather than long-term accumulation effects

$>$ Main focus: Single-Event Effects (SEE)

\section{Main Hardware Components for Analysis}

$>$ Processing Logic $(\mathrm{PL})$ :

7 Sub-Components: BRAM, CRAM

$>$ SEE/day: 3.21

7 Processing System (PL):

7 Sub-Components: OCM, D-CACHE, ALU, FPU, Peripheral

7 SEE/day: 8.22E-02 


\section{Bare Fault Tree Model}

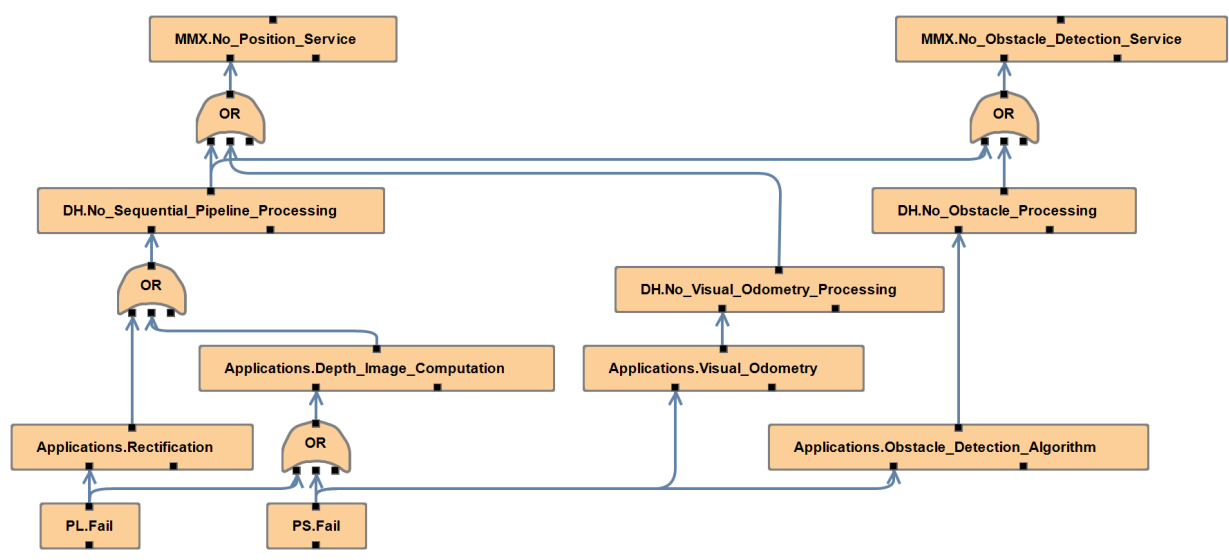

Figure: Bare fault tree model without FDIR 


\section{Summary of Evaluation Results}

Experiment Setup

7 Defined (sensible) configurations of increasing complexity

7 FT size, configuration costs, MTTF, and reliability after 50 days 


\section{Summary of Evaluation Results}

Experiment Setup

7 Defined (sensible) configurations of increasing complexity

$>$ FT size, configuration costs, MTTF, and reliability after 50 days

Results

$>$ Could answer the question if simpler configurations would suffice 


\section{Summary of Evaluation Results}

Experiment Setup

7 Defined (sensible) configurations of increasing complexity

$>$ FT size, configuration costs, MTTF, and reliability after 50 days

Results

$\neg$ Could answer the question if simpler configurations would suffice (sadly: No) 


\section{Summary of Evaluation Results}

Experiment Setup

7 Defined (sensible) configurations of increasing complexity

$>$ FT size, configuration costs, MTTF, and reliability after 50 days

Results

$\neg$ Could answer the question if simpler configurations would suffice (sadly: No)

$>$ Generated fault trees with $\sim 100$ nodes 


\section{Summary of Evaluation Results}

Experiment Setup

7 Defined (sensible) configurations of increasing complexity

7 FT size, configuration costs, MTTF, and reliability after 50 days

Results

$>$ Could answer the question if simpler configurations would suffice (sadly: No)

$>$ Generated fault trees with $\sim 100$ nodes

$>$ Reduced modeling effort by $80 \%$ for most complex configuration 


\section{Conclusion}

\section{Recap}

7 Separated software fault model (Bare model) and mitigation fault models (Fault Tree Library)

7 Reusable

$\neg$ Achieved significant reduction on modeling effort 


\section{Conclusion}

\section{Recap}

7 Separated software fault model (Bare model) and mitigation fault models (Fault Tree Library)

7 Reusable

$>$ Achieved significant reduction on modeling effort

\section{Towards the future}

7 Automatic optimization of configurations? Constraints defining what a "sensible" configuration is?

7 Coupling with code generation 


\section{Conclusion}

Recap

7 Separated software fault model (Bare model) and mitigation fault models (Fault Tree Library)

7 Reusable

$>$ Achieved significant reduction on modeling effort

\section{Towards the future}

7 Automatic optimization of configurations? Constraints defining what a "sensible" configuration is?

$>$ Coupling with code generation

\section{Thank You!! Questions?}

